\title{
Trabajadores de plataformas digitales: Condiciones laborales en plataformas de reparto a domicilio en Argentina
}

Workers of digital platforms: Working conditions in on-demand delivery platforms in Argentina

Andrea Del Bono

Centro de Innovación de los Trabajadores (CITRA-CONICET-UMET), Argentina

delbonoandrea@gmail.com

\section{ReSUMEN:}

El artículo identifica las problemáticas que caracterizan a las condiciones laborales de los repartidores de dos plataformas de reparto a domicilio -la catalana Glovo y la colombina Rappi- que operan desde 2018 en varias ciudades argentinas. A partir de una investigación basada en entrevistas a trabajadores y trabajadoras, análisis de foros y redes sociales, observaciones de campo y relevamiento de prensa se analiza la situación en el empleo de los repartidores, en lo que se refiere a la relación entre la plataforma y su papel como empleador y al reconocimiento de estos en su carácter de trabajadores. A partir de una visión de conjunto, las condiciones laborales se describen como preocupantes porque reflejan el trato injusto que reciben los trabajadores y trabajadoras de plataformas. Se analiza también la naturaleza de la gestión algorítmica y su impacto sobre la organización del trabajo. Finalmente, el artículo se interroga sobre las dificultades que tienen los repartidores para organizarse de manera colectiva y presenta las estrategias que han desplegado los trabajadores de plataformas para hacer oír sus reclamos.

Palabras ClaVe: Trabajo de plataforma, Condiciones laborales, Organización colectiva.

\section{ABstract:}

The article identifies the problems that characterize the working conditions of the delivery workers of two on-demand delivery platforms - the Catalan Glovo and the Colombian Rappi - that operate in several Argentine cities since 2018. Based on interviews with workers, analysis of forums and social networks, field observations and press surveys, the research analyzed the situation in the employment of the delivery workers via apps, in what refers to the relationship between the platform and its role as employer, and their recognition as workers. From an overview, the working conditions are described as worrying because they reflect the unfair treatment received by platform workers. The nature of algorithmic management and its impact on the organization of work is also analyzed. Finally, the article asks about the difficulties that have the delivery workers in organizing collectively and presents the strategies that platform workers have deployed to make their claims heard.

KEYWORDS: Platform work, Working conditions, Collective organization.

\section{INTRODUCCIÓN}

El mundo del trabajo es actualmente testigo del surgimiento y desarrollo de nuevas formas de empleo, muchas de las cuales están vinculadas con las trasformaciones tecnológicas que se vienen produciendo en los últimos tiempos y que encuentran su manifestación más emblemática en el fenómeno de la digitalización de la economía. Aunque se trata de un fenómeno amplio y complejo, quienes caracterizan la "economía digital" destacan como sus principales características la importancia estratégica de la información digitalizada (por su cantidad y diversidad) y la relevancia de la red, como principio organizador por excelencia de la economía y de la sociedad en su conjunto (Valenduc y Vendramin, 2016). La fragmentación global de las cadenas de valor, la interconexión de las capacidades productivas y la difuminación de los límites entre productores, vendedores y consumidores son otras de las características sobresalientes (Fischer y Fuchs, 2015). En el marco de un nuevo modelo de producción industrial y de servicios, mediado por una nueva generación de tecnologías digitales, que permiten aprovechar todo el valor de las redes, surgen nuevos modelos de negocios y nuevos mercados (digitales). Un fenómeno que

Recepción: 15 de julio de 2019 | Aprobación: 17 de septiembre de 2019 | Publicación: 24 de octubre de 2019

Cita sugerida: Del Bono, A. (2019). Trabajadores de plataformas digitales: Condiciones laborales en plataformas de reparto a domicilio en Argentina. Cuestiones de Sociología, 20, e083. https://doi.org/10.24215/23468904e083 
ejemplifica bien esta evolución es la emergencia de plataformas digitales de trabajo, uno de los cambios más importantes en el mundo laboral en los últimos diez años, que ofrece oportunidades para la producción y la prestación de una variedad de servicios que se proporcionan a través de mercados (plataformas) en línea (ILO, 2018).

Una de las señas de identidad de la etapa actual de cambio tecnológico es que su influencia irrumpe en todas las economías, y no sólo en las más desarrolladas, sobre todos los sectores productivos, sean de producción de bienes o de prestación de servicios; alcanzan a los trabajos de menor cualificación pero también a los más cualificados, y afectan a todo tipo de trabajadores bajo cualquier régimen jurídico laboral. En esa dirección hay tendencias, como las del trabajo por internet y las del trabajo en plataformas digitales, que reflejan el fuerte carácter disruptivo de las innovaciones tecnológicas. Hoy en día, es cada vez más sencillo reemplazar la prestación de un servicio tradicionalmente realizada por un trabajador y descentralizarla -en línea- en forma de llamamiento o convocatoria. Este nivel de descentralización permite a las empresas de base tecnológica acceder a través de plataformas en línea a una gran cantidad de personas dispuestas a realizar tareas remuneradas.

Estas formas de trabajo plantean una serie de circunstancias que son motivo de preocupación y que exigen formas apropiadas de regulación, ya que dan lugar a nuevas formas de empleo que combinan lugares de trabajo no tradicionales, el uso de tecnologías -Internet, computadoras y otras herramientas informáticas-, y nuevos arreglos contractuales, generalmente informales y precarios.

Frente a este fenómeno, la literatura especializada plantea una serie de interrogantes acerca de cuáles son las tendencias que pueden considerarse verdaderamente novedosas -y cambios sustanciales en el mundo el trabajo-y cuáles no conllevan una ruptura, sino continuidad y profundización de evoluciones ya existentes en términos de reestructuración productiva y flexibilización laboral (De Stefano, 2016, Valenduc y Vendramin, 2016, Florisson y Mandl, 2018). El debate no es nuevo en absoluto; se planteó también frente a las innovaciones técnicas, de producción y de organización del trabajo de los años ochenta y noventa del siglo pasado, tiene su epicentro en los países más desarrollados, y se reedita frente a la digitalización del mundo del trabajo y al avance de formas de empleo eventuales, informales y precarias, mediadas por el uso de plataformas digitales de trabajo y aplicaciones informáticas (apps).

En los países de América Latina la preocupación por este fenómeno despierta un interés más reciente que transcurre en torno a reflexiones sobre el impacto que el cambio tecnológico produciría en el empleo y sus características, y a la incertidumbre sobre las relaciones laborales resultantes. Dada la participación periférica de la región en el esquema actual de división global del trabajo y considerando el papel hegemónico que adquieren los contextos de marcada desprotección y de ausencia de regulación, las preguntas claves profundizan en la relación entre nuevas tecnologías y nuevas formas de precariedad laboral y en las alternativas de regulación, de formación y de organización, para garantizar la protección de los trabajadores y trabajadoras del desajuste entre las regulaciones y políticas laborales existentes y las nuevas formas de empleo (Bensusán, 2017).

Aunque las plataformas digitales de trabajo adoptan formas diversas, tienen como elemento en común el potencial que demuestran para cambiar la forma en que se organiza y realiza el trabajo, y para alterar la calidad de los empleos. Por más que el trabajo de las plataformas tiene puntos en común con muchas modalidades laborales existentes, la mediación de la tecnología digital da lugar a nuevas formas de trabajo que se caracterizan por el corrimiento de límites en varios niveles - jornada de trabajo, salarios, autonomía, control, etc.-, lo que plantea múltiples preguntas en el campo de la regulación. En este artículo analizamos algunas de esas transformaciones, a partir de la investigación realizada sobre las condiciones laborales de los trabajadores de plataformas de reparto a domicilio.

Según De Stefano (2016), una primera clasificación permite distinguir entre dos tipos de plataformas, las plataformas en línea, en las cuales el trabajo se terceriza on line mediante convocatorias abiertas a una audiencia geográficamente dispersa que trabajará ofreciendo servicios virtuales, y las sustentadas por 
aplicaciones móviles (apps) con geolocalización, en las que el trabajo se asigna a individuos que prestan servicios físicos situados en zonas geográficas específicas. Nuestro análisis se enfoca exclusivamente en esta segunda modalidad.

A partir de una investigación basada en entrevistas a trabajadores y trabajadoras, análisis de foros y redes sociales, observaciones de campo en la ciudad de Buenos Aires y en la ciudad de La Plata, relevamiento de prensa y análisis de documentación empresarial, hemos identificado las problemáticas clave que abarcan a los repartidores de dos plataformas de reparto a domicilio, la colombiana Rappi y la empresa catalana Glovo.

A continuación, presentamos, en primer lugar, algunas reflexiones de carácter conceptual sobre el trabajo de plataformas; analizamos luego la situación en el empleo de los repartidores en lo que refiere a la relación entre la plataforma y su papel como empleador y en lo que refiere al reconocimiento de estos en su carácter de trabajadores. Luego, presentamos las problemáticas más relevantes en lo que a condiciones laborales se refiere, las cuales, vistas en su conjunto, resultan inquietantes, dado que reflejan el trato injusto que reciben quienes trabajan en las plataformas de reparto a domicilio. Analizamos también la naturaleza de la gestión algorítmica, deteniéndonos en algunos de sus impactos sobre la organización del trabajo de reparto. Más adelante, nos preguntamos por las dificultades que tienen los trabajadores de plataformas para organizarse de manera colectiva, y por las chances que tienen para hacer oír sus reclamos. Analizamos entonces la forma en que se han organizado los trabajadores comprendidos en nuestro estudio. Finalmente, presentamos unas reflexiones finales a modo de conclusión.

\section{ECONOMÍAS DE PLATAFORMA: CONSIDERACIONES CONCEPTUALES}

En los últimos años los desarrollos en la tecnología digital han impulsado el surgimiento de plataformas en línea que cumplen la función de poner en contacto la oferta y la demanda de bienes y servicios vinculando directamente clientes con prestadores de servicios individuales. Como han analizado Drahokoupil y Fabo (2016), por esta vía disminuyen los costos de la gestión de mano de obra y del acceso temporal a bienes y servicios gracias a la consolidación de verdaderas plataformas de subcontratación en línea, cuyo desarrollo impacta en los mercados laborales, particularmente en la relación laboral. Esta nueva forma de organizar el trabajo y las actividades comerciales ha sido analizada en el marco de la así llamada "economía colaborativa", entendida como una nueva manera de compartir o intercambiar tanto bienes tangibles como intangibles a través de los nuevos espacios tecnológicos y las comunidades sociales (Vallecillo Gámez, 2017). Sin embargo, la noción de "economía colaborativa” resulta bastante engañosa (Drahokoupil y Fabo, 2016, p. 2), ya que no alcanza a reflejar la tendencia que se registra actualmente, de extensión y amplificación de los mecanismos del mercado, a partir del impacto del cambio tecnológico en la organización del trabajo. Justamente, esa amplificación es la clave del éxito de las plataformas digitales y se manifiesta, por ejemplo, a través de la subcontratación de trabajo colectivo (crowdsourcing), de la división del trabajo en pequeñas tareas independientes de corta duración, y de la externalización del servicio a través de trabajadores autónomos.

Asimismo, otras nociones como las de "economía compartida", "economía a demanda” y "economía de pares", utilizadas con connotaciones positivas como equivalentes de la "economía colaborativa", tampoco aportan más precisión para entender el fenómeno de las plataformas digitales, ya que no son unívocas. Se trata de términos que describen un ámbito más amplio de actividades on line -incluso sin que medie el trabajo remunerado-, que abarcan el comercio de bienes materiales o de capital, así como actividades no comerciales.

En síntesis, las nociones abstractas de colaboración e intercambio no son fértiles para describir los alcances de las actuales mutaciones económicas, cada vez más dependientes de la tecnología digital, del uso de datos y de Internet. La colaboración o la interacción entre pares representa algo diferente de "compartir", y estos términos son evidentemente diferentes, a su vez, del significado asociado a la idea del on-demand (bajo demanda). Asimismo, el concepto de "colaboración" no da cuenta de relaciones que se concretan en el ámbito del mercado y que facilitan el acceso al uso de bienes y servicios. En este sentido, a través de la mayoría de 
las plataformas digitales se desenvuelven transacciones estándar de mercado que bien podrían ser describirse con la idea de "alquilar" en lugar de la de "compartir" (Drahokoupil y Fabo, 2016).

En definitiva, con las plataformas lo que se redefinen son los mecanismos que ponen en contacto oferentes y demandantes de un bien para comercializarlo, no se trata de una actividad nueva, sino de una nueva forma de realizar las transacciones utilizando las nuevas tecnologías y, en numerosos casos, de una actividad de comercio tradicional a la que se ha unido la ventaja de la digitalización. El fenómeno subyacente y que vale la pena poner en relieve es el uso de plataformas digitales en línea, que reducen los costos de transacción de la mano de obra y posibilitan el acceso temporal a bienes y servicios.

Por lo tanto, según la bibliografía consultada, los términos más exactos para analizar estos fenómenos son "economía de plataforma" (platform economy) y "trabajo de plataforma" (platform work) (Florisson y Mandl, 2018, p. 1). Desde 2018, organismos estadísticos de la Unión Europea adoptan la definición de trabajo de plataforma, y entienden por tal "una forma de empleo en la cual las organizaciones o individuos usan una plataforma en línea para acceder a otras organizaciones o individuos para resolver problemas específicos o para proporcionar servicios específicos a cambio de un pago" (Florisson y Mandl, 2018, p. 2). El foco de la atención está puesto en la forma en que las plataformas combinan la oferta y la demanda, utilizando para ello trabajo remunerado. ${ }^{1}$ El trabajo de plataforma y sus características dependen de la forma en que se articulan una serie de elementos, de cómo se produce la interacción entre la plataforma, el cliente y el trabajador, de cómo se produce el desglose del trabajo en mínimas tareas, de la organización de los servicios bajo demanda, y de la forma en que se utiliza la subcontratación y la contratación externa. De estas articulaciones resultan una serie de acuerdos y relaciones laborales, que van desde trabajo temporal, por cuenta propia, pasando por autoempleo y el trabajo informal, hasta el trabajo a destajo y a domicilio. Dependiendo de la situación específica, el trabajo que se proporciona puede ser digital o manual, alcanzar distintos grados de calificación, y exigir una entrega local (en persona) o simplemente una actividad en línea. Las tareas más comunes incluyen: tareas profesionales (por ejemplo, desarrollo de software o diseño gráfico), transporte (por ejemplo, transporte de personas o entrega de alimentos), tareas del hogar (por ejemplo, limpieza), microtareas (por ejemplo, etiquetar imágenes en páginas web).

En cuanto a las actividades que se realizan a través de plataformas digitales de trabajo, también se han utilizado diferentes términos para describirlas. Tal como sintetiza Bensusán (2017, p. 92), nuevos conceptos como el crowdworking (trabajo colectivo o por multitud) y el on-demand work via app (trabajo bajo demanda o a pedido a través de aplicaciones) emergieron con el nuevo siglo, superponiéndose a figuras tradicionales de empleo atípico. Estos empleos asociados con la economía digital y caracterizados por la temporalidad y por su carácter esporádico se conceptualizan actualmente en el marco de la "economía de los pequeños encargos" (gig economy), cuya naturaleza puede involucrar desde tareas muy puntuales o parceladas -microtareas-- hasta otras de mayor contenido. Según argumentan Florisson y Mandl (2018), en este terreno también reina la confusión, y existe una multiplicidad de términos utilizados para describir el trabajo organizado a través de plataformas digitales (gig work, on-demand work, work-on-demand via apps, platform work, digital labor). La clave aquí es que la diferente terminología y una definición deficiente de lo que se abarca en cada término no siempre permite dar cuenta de lo importante: el fenómeno más amplio de la creciente informalidad y precarización de dichos empleos.

\section{Trabajo de Plataformas: ENCUBRIMiEnto de laS RElaCiones DE TRABAJO}

Según acabamos de mencionar, algunas de las trasformaciones actuales del mundo del trabajo se originan y se amplifican a través de plataformas digitales. Se trata de una nueva forma de organización del trabajo, cuyo ejemplo icónico y más polémico es la mundialmente famosa Uber, que conecta prestadores de servicios y usuarios de transporte. Con un crecimiento sostenido también se expanden rápidamente en las 
capitales de todo el mundo las plataformas de cadetería y repartos a domicilio, como las británicas Deliveroo y Just-Eat, la empresa española Glovo, la ampliación de Uber en el sector gastronómico, Uber Eats, entre otras.

En Argentina, las plataformas de delivery, gestionadas por empresas internacionales, como la colombiana Rappi y la española Glovo, comenzaron a operar durante 2018. Aunque la información sobre cantidad de trabajadores es un dato que ambas empresas manejan con confidencialidad, se estima que en la ciudad de Buenos Aires hay cerca de 2.000 repartidores -más de 1.000 "rappitenderos" y cerca de 1.000 "glovers"-, con un promedio de entre 150 y 200 conectados al mismo tiempo (Casas, 2018). También se registra un crecimiento sostenido de repartidores en ciudades como Córdoba, Rosario y La Plata, donde ya operan ambas empresas, con flotas aproximadas de 500 repartidores. Sin embargo, se desconoce el número cierto del total de trabajadores debido al recambio constante con el que se manejan las plataformas.

Ahora bien, lo que resulta clave subrayar es que este tipo de plataformas se está expandiendo velozmente gracias a los beneficios de la "economía bajo demanda", una de las expresiones más extremas de la externalización del trabajo (Ginès i Fabrellas, 2016). Como ha señalado De Stefano (2016, p. 4), en ese contexto la flexibilidad alcanza niveles sin precedentes, los trabajadores ofrecen su trabajo "just-in time" y son retribuidos sobre una base de "pay-as-you-go" (a destajo), es decir, que en la práctica son retribuidos solo durante los momentos en que trabajan para el cliente. Evidentemente, tal mercantilización del vínculo laboral no es exclusiva de la gig economy; se trata más bien de una tendencia que abarca a una parte bien vasta del mercado laboral. No obstante, las características propias de una demanda basada en tareas acotadas y esporádicas exacerban esa tendencia que se traduce en el desplazamiento del trabajo subordinado por el trabajo por cuenta propia.

Los jóvenes trabajadores de las plataformas de envíos a domicilio, que recorren las ciudades argentinas en bicicleta y en moto, son trabajadores formalmente considerados como autónomos, a quienes no se les reconoce relación de dependencia. Con la misma argumentación de la pionera Uber, también Rappi y Glovo se autodefinen como "empresas de tecnología". En ambos casos, las plataformas postulan que son meramente proveedoras de una herramienta informática - una base de datos para conectar clientes con prestadores de servicios individuales- para encubrir cualquier relación de dependencia y sortear el cumplimiento de las protecciones propias de la legislación laboral. La intención de no reconocer las actividades que realizan sus repartidores - "globers" y "rappintenderos" - como trabajo se refleja en el propio léxico que utilizan las empresas para exaltar el emprendedurismo y el autoempleo: "red de consumidores", "red de productores", "contratista independiente", "colaborador", y hasta "microempresario". Mientras tanto, términos como "trabajo" o "trabajadores" se usan raramente en este contexto; se apela constantemente a las ventajas del trabajo virtual eventual ("ganá dinero y seguí disfrutando de tu familia y amigos", "repartí tu tiempo entre tus estudios, trabajo o cualquier otra actividad que hagas") presentado como una especie de limbo o de dimensión paralela en la que se supone que la protección laboral y la regulación del empleo no se aplican, por defecto.

La estrategia local de empresas internacionales como Rappi y Globo no es inédita, replica el modelo de negocio de las plataformas que operan en Europa y EE. UU., donde, por regla, los trabajadores de plataformas de mensajería no gozan de la protección que establece la legislación laboral y mayoritariamente son catalogados como contratistas independientes, trabajadores por cuenta propia, o (falsos) autónomos. En Argentina, los repartidores de las plataformas de delivery están en una situación de mucha vulnerabilidad, a merced de los términos y condiciones de cada plataforma, los cuales, además, "cambian sin previo aviso según la voluntad del empresario" (Manuel, trabajador de Glovo). Las empresas alegan que no hay relación de dependencia y los "glovers" y "rappitenderos" recorren la ciudad desprotegidos sin ningún tipo de seguro, mientras que temas urgentes como "la cobertura de un seguro de salud, la protección en materia previsional, el derecho a descanso, a un salario mínimo, no llegan ni a contemplarse, dado el vacío regulatorio y legal en el que se desarrolla la actividad" (Abogado asesor de la APP).

Ahora bien, diferentes análisis avalan la presunción de laboralidad para los prestadores de los servicios que ofrecen las "empresas de tecnología” (Todolí Signes, 2015; Ginès i Fabrellas, 2016; Bensusán, 2017). 
Según el análisis de Vallecillo Gámez (2017, p. 3), estas empresas prestan un servicio específico por más que se autodefinan como simples bases de datos. Se insertan en un sector concreto, establecen sistemas de retribución, inciden en el precio del servicio, controlan a los prestadores del servicio -establecen tanto procesos de selección como un sistema de retroalimentación de las opiniones de los clientes-, además de fiscalizar al prestador a través de las propias plataformas y de herramientas de geolocalización.

La plataforma fija los precios, la jornada, controla a los trabajadores, la ejecución de la tarea y distribuye premios y castigos, según el rendimiento, en ese marco no es legal considerar a los "rappitenderos" como autónomos, es más, no tengo ninguna duda de que se trata de trabajo en relación de dependencia (Abogado asesor de la APP).

Sin embargo, las plataformas argumentan que no existe supervisión ni control sobre los repartidores. Gracias a las posibilidades que ofrecen las nuevas tecnologías de flexibilizar ciertas notas de subordinación y dependencia jurídica, tanto Rappi como Glovo ofrecen a los jóvenes trabajadores -por mensajes de texto, en la charla de capacitación inicial, e incluso con anuncios de la propia app- que sean "sus propios jefes" y "organicen su propio tiempo", "libertad de movimientos", y la posibilidad de elegir ellos mismos el momento de trabajar ("activate cuando quieras"). Es decir, la supuesta libertad para decidir cuándo y cómo trabajar.

El tema para dilucidar y así sopesar las implicancias que tiene para la protección del trabajo y el empleo la mayor flexibilidad que proporcionan las plataformas en la ejecución del trabajo es si siguen existiendo (o no) expresiones de la subordinación tradicional, entendida como la posibilidad de controlar la actividad del trabajador e ir transmitiendo indicaciones sobre la forma de realizar las tareas (Todolí Signes, 2015). Como ha analizado Bensusán (2017), las plataformas tecnológicas mantienen un rasgo fundamental de la empresa clásica: el control sobre los trabajadores. Recibir evaluaciones negativas de los usuarios, rechazar los pedidos más lejanos, no trabajar en horarios pico o los fines de semana puede resultar en un "bloqueo" del "socio" de la plataforma, lo que en términos reales resulta equivalente a un despido.

Te bloquean y ya no podés trabajar más. No te notifican. Simplemente te dicen que estás inhabilitado y que recurras al sector de comunicaciones que nadie sabe quién es (José, trabajador de Rappi).

Como ha puntualizado Todolí Signes (2015), se trata de un tipo de subordinación en la que los controles de la prestación no han desaparecido, pero se han transformado. No es el empleador quien vigila directamente al trabajador, sino que el control se realiza a través de las evaluaciones del cliente. Las empresas delegan amplias funciones de control en sus clientes y luego utilizan la información que estos generan para tomar decisiones sobre los repartidores. El trabajo de los "glovers" y los "rappitenderos" está controlado por lo que se ha denominado gestión algoritmica (Lee et al., 2015), entendida como un sistema de ajustes en el trabajo en el que los trabajos humanos se asignan, optimizan y evalúan mediante algoritmos y rastreo de datos. Mientras el usuario de un repartidor está activado la app asigna pedidos cercanos, el sistema funciona mediante un rastreo de la proporción de pedidos aceptados en determinado lapso y realiza un promedio de la puntuación que los clientes asignaron al repartidor después del servicio. Los repartidores pueden empeorar su calificación por no aceptar suficientes pedidos o por bajas puntuaciones; reciben incentivos para trabajar durante determinadas horas y mensajes que los animan a esforzarse más en los momentos de picos de demanda.

Sacan promociones todo el tiempo, por ejemplo, mil pesos si en la semana conseguís hacer cinco pedidos en tres horas (María, trabajadora de Glovo).

Se observa entonces que si bien los repartidores pueden decidir sobre aspectos importantes de su trabajo -extensión de la jornada, horarios, descansos, etc.-, no dejan por eso de estar insertos en un sistema que organiza la actividad que realizan y la retribución que reciben, del que incluso pueden ser "despedidos" cuando la calidad de su trabajo no alcanza los parámetros establecidos. Esta situación lleva a plantear la existencia de vínculos de subordinación jurídica, económica y técnica, vínculos que plataformas como Rappi y Glovo logran difuminar con la gestión algorítmica. 
A pesar de que estas circunstancias han tenido bastante difusión, el debate que se plantea en muchos países del mundo en relación con los problemas de los empleos de las plataformas digitales apenas se inicia en el caso argentino, donde los poderes públicos vienen mostrando falta de reacción para regular el funcionamiento de las plataformas y se carece de jurisprudencia al respecto. Por ejemplo, volviendo al caso de Uber, en ciudades como Nueva York y Paris existe regulación de la actividad -aunque todavía con sentencias y pronunciamientos contradictorios-, la cual iguala a este tipo de transporte con los taxis en materia de regulación laboral y salarial para los conductores (Auvergnon, 2016). Lo mismo ocurre desde diciembre de 2018 en el Reino Unido, donde la empresa perdió un recurso presentado contra la decisión judicial que había otorgado derechos laborales a sus conductores. En relación con las plataformas de reparto a domicilio, el debate no se encuentra zanjado aún y la justicia emite fallos contradictorios en el Reino Unido, en Francia, en Italia y en España, en los que se reconoce a los trabajadores en distintas sentencias como autoempleados, o como trabajadores dependientes de la empresa.

\section{Trabajo Bajo DEMANDA Y gestión ALgorítmica: CARACTERÍSTICAS DEFINITORIAS DE LA RELACIÓN LABORAL PRECARIA}

En Argentina, según datos del Ministerio de Trabajo, los pequeños trabajadores independientes monotributistas- son el componente de empleo de más rápido crecimiento interanual frente al empleo total. Entre agosto de 2017 y el mismo mes de 2018 los monotributistas puros aumentaron un 6,8 \%, la ocupación total registrada creció solamente un 1,8 \%, y los trabajadores asalariados que aportan al Sistema Integrado Previsional Argentino (SIPA) aumentaron apenas un 0,5 \% (González Cao, 2018, p. 8). Esta tendencia nada positiva- refleja el reemplazo de relaciones permanentes de empleo en relación de dependencia por otras formas eventuales y desprotegidas. En este terreno fértil se expanden las plataformas de trabajo que prestan servicios de baja calificación -como Rappi y Glovo- y que operan con contrataciones precarias de ciertas poblaciones desempleadas e inactivas que no logran acceder a buenos empleos.

En los casos de las plataformas de reparto a domicilio, la población vulnerable que conforma las cuadrillas de repartidores cuenta con trabajadores y trabajadoras jóvenes, un segmento de la población activa en el que el aumento de la desocupación crece a mayor ritmo. Según el Instituto Nacional de Estadísticas y Censos (INDEC), la Argentina cerró 2018 con un aumento interanual de casi dos puntos de la desocupación, del $9,1 \%$, y un deterioro de todos los indicadores de empleo. El desempleo afecta más a los jóvenes argentinos que a los adultos. La tasa de desempleo de los jóvenes más que duplica a la de la población adulta en general y viene ampliándose desde 2004, por lo que Argentina es en la actualidad el país con mayor desempleo juvenil de la región. Más allá del desempleo, otro problema tanto o más serio entre los jóvenes es la informalidad; seis de cada diez jóvenes que trabajan lo hacen de forma precaria, lo que se traduce en que a menudo ganan el salario mínimo o menos, trabajan en jornadas extendidas en situaciones insalubres y en situaciones de mucha desprotección (Paugan, 2007).

Para profundizar aún más este cuadro de informalidad y precariedad, las plataformas que operan en las grandes ciudades de Argentina también sacan ventaja de la difícil situación de la inmigración venezolana. En un éxodo sin precedentes, una gran cantidad de venezolanos llega cada semana a la Argentina, muchos de ellos con un buen nivel de estudios. Con un certificado de residencia en trámite, los jóvenes venezolanos reparten currículums pensando en poder trabajar de lo suyo, mientras realizan todo tipo de trabajo informal. En la ciudad de Buenos Aires buena parte de los "glovers" y "rappintenderos" son inmigrantes venezolanos ("hoy el consulado venezolano son Rappi y Glovo") que trabajan largas jornadas para poder cubrir deudas básicas como alquiler y comida, y que tienen como objetivo final poder ahorrar algún dinero para mandar a su familia en Venezuela (Ayzaguer, 2018).

En un contexto recesivo como el que atraviesa Argentina, los jóvenes y los inmigrantes buscan trabajo en las plataformas de reparto, las cuales no exigen demasiadas condiciones. Los requerimientos son la presentación 
del documento de identidad, ser mayor de 18 años, tener un lugar de residencia comprobable y asistir a una charla de capacitación. Aquellos que llegan a incorporarse como repartidores tienen que realizar su inscripción fiscal al régimen de Monotributo para facturar los servicios prestados. Como monotributista, es el propio trabajador quien asume con sus aportes la obra social, la seguridad social y la aseguradora de riesgos laborales (ART), eximiendo de estas cargas a las plataformas de trabajo.

Las empresas deciden las tarifas, las comisiones y nos hacen tributar por las operaciones comerciales por las que ellos deberían ser responsables(Javier, trabajador de Rappi)

Según pudimos constatar, esta no es la única circunstancia que refleja el trato injusto que reciben los repartidores de las plataformas de delivery; otras situaciones reflejan arbitrariedades, inseguridad y desprotección.

En los casos analizados, existe una serie de requisitos que ponen a los trabajadores en la situación injusta de tener que pagar -ellos mismos- a las plataformas para comenzar a trabajar. El primer día, los "glovers" alquilan el uso de la app y los "rappitenderos" abonan (por única vez) la caja de telgopor para trasladar los pedidos. Recién después de cumplir con quince entregas satisfactoriamente la empresales vende los uniformes. Según se observa, los propios trabajadores responden por los gastos iniciales, a lo que se agrega la inversión en una bicicleta -o en una moto- cuando no cuentan con vehículo propio.

Vamos por la ciudad haciéndoles publicidad gratis, con las cajas y los uniformes con el logo de Rappi que nosotros mismos les compramos, poniendo dinero de nuestro bolsillo (José Antonio, dirigente gremial de Rappi).

Una vez en la calle, los repartidores encuentran condiciones laborales caracterizadas por la desprotección y la inseguridad, que han sido denunciadas reiteradamente y reconocidas por las autoridades de los municipios en los que operan las plataformas. En la ciudad de La Plata, los "glovers" denunciaron frente a la empresa y en los medios de comunicación múltiples hechos de violencia y de inseguridad. Robos de la recaudación a punta de pistola captados por las cámaras de seguridad de los edificios, el robo de sus motos, y el grave incidente en el que resultó baleado y hospitalizado un repartidor en un asalto. ${ }^{2}$ La única medida de seguridad con la que cuentan los repartidores son las alertas en sus propios grupos de WhatsApp.

Tenemos un grupo de WhatsApp grandísimo; somos como trescientos, lo fuimos armando por la calle, cuando nos encontramos. Tengo otro grupito, somos veinte y pico, todas chicas; nos avisamos los pedidos que tomamos, estamos siempre atentas y nos cuidamos entre nosotras (María, trabajadora de Glovo).

En la ciudad de Buenos Aires, la desprotección laboral de los “rappitenderos" se manifestó con la muerte de un joven repartidor de 20 años, que fue atropellado por un camión cuando se dirigía a entregar un pedido en bicicleta. ${ }^{3}$ Pocos días antes del accidente, un fallo de la justicia de la ciudad de Buenos Aires había puesto la atención en las condiciones de trabajo de los repartidores, cuando dictaminó que se debía impedir el funcionamiento de las plataformas (Rappi, Glovo y Pedidos Ya) hasta que las condiciones mínimas de seguridad establecidas por el Código de Transporte de la ciudad estuviesen aseguradas (cascos, luces, cajas de reparto adecuadas, y seguro de vida y accidentes). Aunque el fallo remarca que la responsabilidad por el cumplimiento de esas medidas de seguridad es de las empresas, las plataformas descargaron estas obligaciones sobre los trabajadores, que resultaron ser los verdaderos perjudicados por la suspensión del funcionamiento de la $a p p$.

Sin dudas, esta no es la solución que los trabajadores buscamos, como así tampoco la prohibición de nuestro trabajo, que es nuestra fuente de ingresos. Las empresas tienen que hacerse cargo de cumplir con las normativas laborales y de tránsito que las leyes establecen (APP/@AppSindical).

Estas situaciones que venimos reseñando responden a los cambios actuales que provoca la digitalización del mundo del trabajo, muy especialmente en lo que hace a la ampliación de los márgenes de flexibilidad -sin 
seguridad-, y al desdibujamiento de los vínculos laborales. Otro de los terrenos en que se manifiesta dicha flexibilidad es el salarial, con los bajos ingresos de los trabajadores.

La experiencia de las ciudades europeas, y más recientemente el de las latinoamericanas, demuestra que los ingresos de los repartidores de plataformas son muy bajos - como se evidencia, por ejemplo, en los casos de Glovoy de la británica Deliveroo-, y que no están garantizados, ya que generalmente sus trabajadores cobran por comisión. Aunque las jornadas de trabajo se alarguen, no siempre se consigue un ingreso suficiente para vivir dignamente (Eurofound, 2018).

En las ciudades argentinas en las que operan, las plataformas pagan a los repartidores solo por cada pedido que llega efectivamente al cliente, y no ofrecen un sueldo básico. Cuando un cliente hace una compra abona a través de la aplicación el precio del producto más el envío, el $100 \%$ del costo del envío pertenece al repartidor y las plataformas reciben de los comercios adheridos una comisión por cada venta. Por cada viaje se generan estimativamente 50 pesos para el repartidor (1,12 USD), valor que puede aumentar en horarios de alta demanda o ante situaciones específicas. Los ingresos exactos son difíciles de estimar, ya que varían según el tipo de servicio, los días de la semana, las horas pico, la ciudad, el rankig de los repartidores, y las tarifas y pagos variables que fijan unilateralmente las empresas. Aproximadamente, los repartidores de las ciudades de Argentina completan un ingreso de entre \$18.000 (406 USA) y \$20.000 (451 USA) al mes. Para alcanzar esa cifra, se trabajan jornadas extendidas de más de ocho horas, feriados y fines de semana.

Trabajan diez horas, doce, muy por encima de los límites legales para alcanzar un ingreso digno (Abogado asesor de la APP).

A pesar de que esos ingresos son bajos, este factor no es el principal motivo de disconformidad de los repartidores. Centralmente, los testimonios que hemos podido recoger reflejan un fuerte rechazo por la "gestión algorítmica". Si bien muchos de los jóvenes repartidores se ven inicialmente seducidos por la idea de autogestionarse y por la posibilidad de organizar su propio tiempo de trabajo articulándolo con otras obligaciones, rápidamente ven reducirse esos márgenes de autonomía.

El hecho es que en las plataformas digitales la gestión algorítmica va mucho más allá de programar y dirigir el trabajo, y pasa a controlar prácticamente todas las dimensiones del acto de trabajar. Möhlmann y Zalmanson (2017) han presentado cinco características de la gestión algorítmica: seguimiento continuo del comportamiento de los trabajadores; evaluación constante de su desempeño, de las evaluaciones del cliente, y de la aceptación o rechazo que estos tienen del trabajo realizado; implementación automática de decisiones sin revisión humana; interacción de los trabajadores con un "sistema" que los priva de oportunidades de retroalimentación, discusión y negociación, y un último elemento de importancia central, la baja transparencia. Según estos autores, la baja transparencia se deriva de las prácticas comerciales competitivas que evitan que las plataformas revelen cómo funcionan los algoritmos, pero también de la naturaleza adaptativa de los algoritmos, según la cual las decisiones cambian de acuerdo con la recopilación datos. Como explican Möhlmann y Zalmanson, las empresas rara vez están motivadas a revelar los criterios de apoyo de sus algoritmos, y a veces no pueden explicar completamente los resultados, lo que da lugar a un sistema muy opaco. En los casos que estamos analizando, el marco de baja transparencia existente se utiliza para promover la rotación de los repartidores, que, cansados de circular todo el día y no recibir pedidos, buscan otro destino laboral.

Cuando recién empezás recibís muchos más pedidos y te entusiasmás, pero cuando llevas varios meses te llegan cada vez menos (...) aunque esperes con la bici en la puerta del McDonald's (...), la propia App elige a los repartidores que recién empiezan. Tenés que irte vos mismo porque ya no ganás dinero (Guillermo, trabajador de Glovo).

“Glovers” y "rappitenderos"describen que la transparencia de la gestión algorítmica es extremadamente baja, lo cual se condice con las experiencias de otros trabajadores de plataformas. La opacidad del funcionamiento de las apps es parte de un nuevo modelo de negocio que ha reescrito las reglas de la competencia, con la estrategia de "el ganador se lleva todo" (Valenduc y Vendramin, 2016). La nueva 
generación de tecnologías digitales genera una cantidad de datos sin precedentes, que constituyen una herramienta clave para la gestión ultraflexible del factor trabajo. Esta gestión apunta a conseguir altísimos niveles de rotación y de renovación de "socios" para sostener agresivas dinámicas competitivas orientadas a desbancar competidores.

En síntesis, los elementos que acabamos de presentar permiten evidenciar el desgaste de los repartidores de plataformas, provocado por la continua evaluación y calificación del desempeño, los mecanismos competitivos de asignación de trabajo, los riesgos del trabajo en las calles, la inseguridad laboral, y la incertidumbre en cuanto a los pagos y los bajos salarios. Desde luego, es importante subrayarlo, estas circunstancias no son exclusivas de los trabajadores y trabajadoras de la economía de plataformas. En todo caso, coinciden o se parecen a distintas formas precarias de trabajo, con la única diferencia de que hacen uso de la herramienta digital. Puede argumentarse, entonces, que la dinámica que generan las plataformas de trabajo es solo una parte de una tendencia más amplia de "eventualización" de la mano de obra (De Stefano, 2016).

\section{Organización de los trabajadores COMO FRENO A LA PRECARIZACIÓN DEL TRABAJO DIGITAL}

Las formas de trabajo de la gig-economy, en las que los rankings y las calificaciones desempeñan un papel importante para garantizar mejores chances, condiciones laborales, e incluso la permanencia en la propia plataforma, imponen un límite al ejercicio de los derechos laborales. En esas circunstancias el ejercicio de cualquier derecho colectivo puede traducirse en un perjuicio para los propios trabajadores. La posibilidad de ser "despedido" fácilmente mediante la simple desactivación de una plataforma -o aplicación- puede magnificar el temor a represalias, por lo tanto, ese tipo de temor se asocia a los trabajos puntuales, esporádicos, eventuales, y bajo demanda de la era digital. Asimismo, con la conectividad permanente que permiten las TICs, las plataformas de trabajo tienen las condiciones para monitorear y desalentar cualquier intento de organización o activismo. Sin embargo, los trabajadores y trabajadoras vienen utilizando una amplia gama de estrategias para promover instancias de acción colectiva, garantías para la libertad de asociación, y avances en la regulación efectiva de su trabajo.

En la mayoría de los casos los conflictos colectivos emergentes en las plataformas de servicios encuentran su impulso lejos de la participación de los sindicatos de clase. En relación con las plataformas de reparto a domicilio, hemos identificado como experiencia pionera la organización de los trabajadores de la empresa Deliveroo con la huelga que tuvo lugar en Londres durante el verano de 2016, que se extendió por distintas localidades del Reino Unido, y a la que se plegaron los trabajadores de UberEasts. La huelga de Londres comenzó a partir de la movilización informal de los trabajadores cuando se les informó que sus contratos pasarían de contemplar un sueldo por hora a un sistema de trabajo a destajo (pago por entrega). El éxito de la protesta obligó a la compañía a permitir que los repartidores pudieran elegir la estructura de pagos y sentó las bases para los futuros litigios. Un año más tarde, los reclamos traspasaron las fronteras británicas y los repartidores fueron a la huelga en más de diez ciudades del Reino Unido, Italia, Francia, España y Alemania. Como parte de ese movimiento se destaca la organización de los trabajadores españoles, representados por colectivos como Riders X Derechos, que ha producido manifestaciones importantes por el mejoramiento de las condiciones de trabajo en las plataformas Glovo y Deliverooy una huelga en julio de 2018, con un alto impacto en ciudades como Barcelona, Valencia y Madrid.

En América Latina, la organización de los trabajadores de plataformas de la ciudad de Buenos Aires también constituye una experiencia pionera, por sus alcances y por haber desembocado en la creación de una organización sindical que agremia a los trabajadores de plataformas tecnológicas y que es la primera de este tipo en la región. Se trata de la Asociación de Personal de Plataformas (APP), que fue impulsada por los trabajadores de Rappi y que aspira a representar a todos los trabajadores dedicados a transportar productos y personas -Glovo, Uber, Cabify, entre otros-. La precariedad de las condiciones laborales de los repartidores, 
los bajos salarios, la inseguridad, la falta de seguridad social, las extensas jornadas son algunos de los factores que estuvieron en la base de la primera huelga de trabajadores de plataformas, que se extendió durante un par de horas - con el bloqueo de la aplicación- la noche del 15 de julio de 2018, y que protagonizaron los trabajadores de Rappide la ciudad de Buenos Aires.

A partir de esa primera experiencia colectiva, que resultó exitosa en términos de adhesión y de impacto en redes sociales y medios de comunicación, frente a la falta de respuesta a sus reclamos (entre otros puntos, aumento del valor del viaje, cese de los "bloqueos" de los repartidores, formalización de trabajadores) y a la inacción estatal en el terreno de la regulación de la actividad, los repartidores profundizaron en la estrategia de la organización colectiva con la creación de la APP.

La mayoría de los trabajadores tenían reclamos semanales y ninguno era escuchado, Rappi nos cerró los canales para comunicarnos y no tuvimos intervención de las autoridades de la ciudad en las denuncias públicas. Por eso decidimos crear la asociación. Vimos en asociarnos la única vía para ser escuchados (Micaela, dirigente gremial de Rappi).

En otras ciudades, como Rosario y La Plata, los trabajadores de Glovo también avanzaron en su organización, en el primer caso, con protestas frente a las oficinas de la empresa, y en La Plata, con protestas y cortes de calle frente a la municipalidad. Los "glovers" planteses también realizaron una medida de fuerza contra la plataforma. La campaña comenzó en las redes sociales y cientos de jóvenes se sumaron a la propuesta de boicotear la aplicación la noche del sábado 30 de marzo de 2019. Los reclamos apuntan a acotar la situación de extrema flexibilidad que venimos reseñando: cambio en las modalidades de contratación, aumento de la tarifa base y por kilómetro, mayor seguridad y estabilidad laboral, entre otras reivindicaciones.

En todos los casos reseñados, frente a los avances de la organización de los trabajadores contra la precarización del trabajo digital, las empresas respondieron con estrategias similares: con medidas de "persuasión", anunciando a través de sus apps aumentos y promociones para desactivar las medidas de fuerza, y con hostigamiento hacia los trabajadores, bloqueando a quienes se organizaron para reclamar derechos mínimos. En la ciudad de Buenos Aires, Rappibloqueó a todo el grupo de trabajadores de la Comisión Directiva de la APP, que habían liderado la actividad sindical. Frente al reclamo de los trabajadores, el Juzgado Nacional de Primera Instancia del Trabajo Nro. 37 ordenó, en un fallo sin antecedentes en el país y en la región, la reincorporación del grupo de trabajadores despedidos y el cese en forma urgente de la conducta antisindical desplegada por RAPPI ARG. S.A.S. De esta manera, el fallo otorga verosimilitud al hecho de que los repartidores trabajan "a pedido" de las aplicaciones y reconoce el derecho al ejercicio de la libertad sindical de los trabajadores de plataformas digitales.

Según nuestro análisis, estas primeras acciones representan un avance que debe valorarse a la luz de las dificultades que plantea la organización de los trabajadores en el entorno de las plataformas digitales. Como analizan Johnston y Land-Kazlauskas (2018), en estos ámbitos la acción colectiva se ve limitada por un abanico de restricciones legales, que acotan profundamente las posibilidades de agremiación y acción sindical y que terminan profundizando la soledad que caracteriza a los mercados laborales digitales, conformados por trabajadores precarizados, que trabajan de forma aislada o en áreas geográficamente dispersas, y que muchas veces compiten entre ellos. Estas características inhiben los esfuerzos de organización colectiva por las grandes limitaciones que entrañan para motivar el compromiso de los trabajadores. Como respuesta, los trabajadores de plataformas y sus organizaciones ensayan los mejores métodos para organizarse en entornos laborales mediados por las tecnologías digitales. En ese entorno, "glovers" y "rappitenderos" han desplegado una "estrategia virtual" (Scasserra, 2019), promovida desde teléfonos celulares y redes sociales, para superar las dificultades que plantea el bajo nivel de conocimiento que existe entre los trabajadores, y para responder al desafío de sostener una acción gremial que funcione como un canal de comunicación y como una herramienta para construir instancias de solidaridad colectiva. 


\section{Conclusiones}

Hasta aquí hemos analizado algunas de las problemáticas que mejor caracterizan a las condiciones laborales de los trabajadores de plataformas de reparto a domicilio, desde que empresas exitosas internacionalmente -como Rappi y Glovo- comenzaron a operar en varias ciudades argentinas. En términos amplios, hemos argumentado que el auge de la economía digital tiene un carácter disruptivo sobre el futuro del trabajo y del empleo. La digitalización, cuando potencia canales de comercialización como el de las plataformas digitales, promueve nuevas formas de organizar y gestionar el trabajo en las que la conexión entre el productor y el consumidor se realiza sin intermediarios gracias a mecanismos de búsqueda muy eficientes que conectan con precisión las partes. Gracias a la "algoritmización" de los nuevos mercados digitales, las plataformas operan con costos prácticamente nulos, con un aumento de eficiencia que permite una importante reducción de costos en los más variados sectores de la economía, muy especialmente, en el terreno de los costos laborales.

Las nuevas formas de trabajo en la economía digital se caracterizan por la desregulación y el corrimiento de límites en varios niveles. En el caso del trabajo de plataformas de reparto, las condiciones de trabajo asociadas con esta forma de empleo incluyen horarios de trabajo muy flexibles, salarios extremadamente variables y altas expectativas en términos de disponibilidad, así como baja seguridad laboral, pocas oportunidades para ascender en la escala salarial y bajas tasas de satisfacción laboral. Los nuevos trabajos de las plataformas estudiadas son una expresión tangible de un nuevo tipo de acoplamiento entre contratos de trabajo y tiempo de trabajo, y entre contratos laborales y protección de los trabajadores, que obligan a plantear nuevas formas de regulación de las relaciones laborales.

Según hemos visto, el funcionamiento de las plataformas digitales se aleja bastante de las bondades de la "economía colaborativa". Los nuevos modelos flexibles de producción de la economía digital (cocreación entre pares) dan lugar a ambigüedades en torno a lo que es genuinamente compartir, y lo que representa aprovecharse del trabajo asalariado no remunerado. En este contexto, la falta de distinción clara del estatus de los trabajadores empleados por las plataformas de reparto a domicilio, entre el trabajador dependiente y el trabajador autónomo, resulta un tema particularmente preocupante por lo que representa en cuanto a la precarización de las condiciones de trabajo y de empleo.

Otro elemento que hemos destacado es la importancia que tiene para los repartidores - "glovers" y "rappitenderos"- su autonomía y el control que desearían tener sobre su trabajo. Según hemos visto, son las propias plataformas las que incumplen con estos requisitos de libertad -que ellas mismas ofrecenmediante la introducción de acuerdos ultraflexibles de trabajo basados en la combinación del big data con la geolocalización. Flexibilidad y autonomía no dan lugar a una articulación virtuosa en el entorno de las plataformas de reparto a domicilio. Mientras que los repartidores hacen malabarismos con sus tiempos de trabajo para obtener buenas valoraciones, la gestión algorítmica produce cierta sobrecarga de trabajo, y el trabajo en la calle produce riesgos para la salud y la seguridad que carecen de visibilidad (accidentes, asaltos, clientes agresivos, etc.). Como el trabajo de los repartidores está organizado exclusivamente a través de las plataformas, los trabajadores no cuentan con canales para transmitir dichas situaciones a las empresas. La combinación de todos estos elementos, a los que se suma la realización de un esfuerzo que no se ve recompensado con un ingreso decente, termina disipando las expectativas iniciales de los jóvenes trabajadores de plataformas.

En este contexto de retroceso de las relaciones laborales tradicionales, en el que las propias plataformas digitales actúan como intermediarios del mercado laboral, hemos analizado algunas de las acciones y acuerdos colectivos - con nuevos modelos de organización sindical- a través de los cuales los trabajadores responden a la amenaza de la dispersión y de la individualización. Con una variedad de estrategias, los trabajadores se organizan para fortalecer su representación y su capacidad de acción en el entorno de la economía de plataformas. La sindicalización, las protestas con bloqueo de las aplicaciones, los foros en línea y el uso de redes sociales representan iniciativas destinadas a fomentar tanto derechos como la propia comunicación entre los 
trabajadores, además de generar conciencia sobre la necesidad de contar con un marco legal para mejorar las condiciones laborales y promover los intereses de los trabajadores y las trabajadoras de plataformas.

\section{ReFERENCIAS}

Auvergnon, Ph. (2016). Angustias de uberización y retos que plantea el trabajo digital al derecho laboral. Revista Derecho Social y Empresa, 6, 1-18.

Ayzanguer, M. (20 de agosto de 2018). Los venezolanos coparon los servicios de mensajería en bicicleta. La Nación. Recuperado de: https://www.lanacion.com.ar/sociedad/los-venezolanos-coparon-los-servicios-de-mensajeria-e n-bicicleta-nid2162967

Casas, X. (27 de abril de 2018). Guerra de delivery boys en la Ciudad ante el aumento de apps de envíos a domicilio. El Cronista. Recuperado de: https://www.cronista.com/negocios/Guerra-de-delivery-boys-en-la-Ciudad-anteel-aumento-de-apps-de-envios-a-domicilio-20180427-0014.html

Bensusán, G. (2017). Nuevas tendencias en el empleo: retos y opciones para las regulaciones y políticas del mercado de trabajo. En G. Bensusán, W. Eichhorst, J. M. Rodríguez (Ed.). Las transformaciones tecnológicas y sus desafios para el empleo, las relaciones laborales y la identificación de la demanda cualificaciones (pp. 81-171). CEPAL: serie Documentos de Proyectos. Recuperado de https://www.cepal.org/es/publicaciones/42539-transformaciones-t ecnologicas-sus-desafios-empleo-relaciones-laborales-la

De Stefano, V. (2016). The rise of the 'just-in-time workforce': On-demand work, crowdwork and labour protection in the 'gig-economy'. International Labour Office (Conditions of work and employment series; Nro. 71). Geneva: International Labour Office.

Drahokoupil, J. \& Fabo, B. (2016). The platform economy and the disruption of the employment relationship. ETUI Policy Brief European Economic, Employment and Social Policy, 5, 1-6.

Eurofound (2018). Employment and working conditions of selected types of platform work. Luxembourg: Publications Office of the European Union. Recuperado de https://www.eurofound.europa.eu/publications/report/2018/e mployment-and-working-conditions-of-selected-types-of-platform-work

Florisson, R. \& Mandl, I. (2018). Digital age. Platform work: Types and implications for work and employmentLiterature review. Luxembourg: Eurofound.

Fisher, E. \& Fuchs, C. (Ed.) (2015). Reconsidering value and labour in the digital age. England: Palgrave-Macmillan.

Ginès i Fabrellas, A. (2016). Crowdsourcing sites y nuevas formas de trabajo. El caso de Amazon Mechanical Turk. Revista Derecho Social y Empresa, 6, 1-20.

González Cao, R. (2018). Los trabajadores de plataforma y la evolución de las relaciones laborales. Documento de Trabajo, CEAT. Buenos Aires: Facultad de Ciencias Económicas.

ILO (2018). Digital labour platforms and the future of work: Towards decent work in the online world. Geneva: International Labour Office. Recuperado de https://www.ilo.org/wcmsp5/groups/public/---dgreports/---dco $\mathrm{mm} /$---publ/documents/publication/wcms_645337.pdf

Johnston, H. \& Land-Kazlauskas, C. (2018). Organizing On-Demand: Representation, Voice, and Collective Bargaining in the Gig Economy. Geneva: International Labour Office. Recuperado de https://www.ilo.org/wcmsp5/group s/public/---ed_protect/---protrav/---travail/documents/publication/wcms_624286.pdf

Lee, M. K., Kusbit, D., Metsky, E. \& Dabbish, L. (2015). Working with machines: The impact of algorithmic and datadriven management on human workers. Association for Computing Machinery (ACM) Conference on Human Factors in Computing Systems (CHI), 1603-1612.

Möhlmann, M. \& Zalmanson, L. (2017). Hands on the Wheel: Navigating Algorithmic Management and Uber Drivers' Autonomy. Thirty Eighth International Conference on Information Systems, 1-17.

Scasserra, S. (2019). El despotismo de los algoritmos. Cómo regular el empleo en las plataformas. Nueva Sociedad, 279, 133-140.

Paugam, S. (2007). Las formas elementales de la pobreza. Madrid: Alianza. 
Todolí Signes, A. (2015). El impacto de la Uber Economy en las relaciones laborales: los efectos de las plataformas virtuales en el contrato de trabajo. IUSLabor, 3, 1-25.

Valenduc, G. \& Vendramin, P. (2016). Work in the digital economy: sorting the old from the new. Working Paper ETUI. Brussels: European Trade Union Institute.

Vallecillo Gámez, M. (2017). Economía colaborativa y laboralidad: Los cabos sueltos entre el vacío legal y la dudosa legalidad. El Futuro del trabajo que queremos. Conferencia Nacional Tripartita, 2, 461-473.

\section{Notas}

1 Como el principal bien comercializado es la mano de obra, no el material o el capital en sí mismo, las plataformas de ventas (como eBay) o las plataformas en las que se proporciona acceso al alojamiento (como Airbnb), así como los servicios financieros, quedan fuera del alcance de lo que entendemos como trabajo de plataforma. Asimismo, las transacciones no comerciales (como voluntariado, redes, redes sociales como LinkedIn) o cualquier otra forma de transacción que no implique pago, no se consideran una plataforma de trabajo.

2 "Me acaban de robar la moto, el dramático audio de otro glover platense asaltado", en 0221.COM.AR, 7 de enero de 2019. Disponible en: https://www.0221.com.ar/nota/2019-1-7-22-13-0--me-acaban-de-robar-la-moto-me-quiero-ma tar-el-dramatico-audio-de-otro-glover-platense. "Habló Kevin, el glover baleado: fue un golpe duro, mi familia todavía lo está procesando”, en 0221.COM.AR, 1 de diciembre de 2018. Disponible en: https://www.0221.com.ar/nota/2018 -12-1-19-20-0-hablo-kevin-el-glover-baleado-fue-un-golpe-duro-mi-familia-todavia-lo-esta-procesando

3 Para seguir estos acontecimientos, y muchos otros que motivan el reclamo de los trabajadores, hemos realizado un seguimiento exhaustivo de la cuenta: @AppSindical (Asociación de Personal de Plataformas). 